\title{
Chemical Evolution in Hierarchical Clustering Scenarios
}

\author{
Patricia B. Tissera \\ Institute of Astronomy and Space Physics, CONICET, Argentina \\ Cecilia Scannapieco \\ Institute of Astronomy and Space Physics, CONICET, Argentina
}

\begin{abstract}
We present first results of an implementation of chemical evolution in a cosmological hydrodynamical code, focusing the analysis on the effects of cooling baryons according to their metallicity. We found that simulations with primordial cooling can underestimate the star formation rate from $z<3$ and by up to $\approx 20 \%$. We constructed simulated spectra by combining the star formation and chemical history of galactic systems with spectral synthesis models and assess the impact of chemical evolution on the energy distribution.
\end{abstract}

\section{Introduction}

The current cosmological paradigm predicts a hierarchical building up of the structure. This scenario is largely supported by observations in the local and high redshift Universe. However, few but important descrepancies remain to be explanied such as the inner most distribution of dark matter in small haloes, the origin of the high baryonic content and metallicity of the intercluster medium, etc. (Shanks 2004). Most of these open problems require comprehensive understanding of galaxy formation, including all the involved scales. Numerical simulations have played an important role in understanding and proving the connection between theory and observations at different scales. However, the modelling of small scale physical processes such as gas cooling, star formation and chemical evolution within a cosmological framework presents different difficulties because of our poor understanding of the relevant physics and numerical limitations. In particular, only recently chemical evolution has started to be treated within cosmological hydrodynamical simulations (Yepes et al. 1998; Cen \& Ostriker 1999; Mosconi et al. 2001).

The dramatic build up of observational evidence on the chemical properties of the stellar population and interstellar medium in the Universe has proved to have a significant impact on the study of galaxy formation. Chemical elements are produced in the stellar interior and ejected in a pacific or violent way after characteristic timescales determined by stellar evolution. Afterwards, these elements can be mixed up by mergers and interactions. It is also important to notice that the metal production is directly linked to the star formation history which can also be affected by the environment (Barton et al. 2000; Lambas et 
al. 2003; Balogh et al. 2004). As a consequence, it is expected that chemical patterns store information of the process of galaxy formation (Freeman \& Bland-Hawthorn 2002).

Another important motivation to include a chemical treatment in galaxy formation models is related to the fact that baryons cool according to their metallicity. Sutherland \& Dopita (1993) showed that a gas cloud with solar metallicity cools at a rate of up to few orders of magnitude more efficiently than a primordial gas cloud. Hence, the enrichment of the interstellar medium can have dynamical consequences and affect the formation of the structure.

In this work, we present first results of a detailed treatment of chemical evolution in Gadget2 (Springel \& Hernquist 2002) based on the implementation of Mosconi et al. (2001). In Section 2 we briefly summarize the main important aspects of the chemical code. Section 3 shows the first results and Section 4 presents the conclusions.

\section{The model}

The chemical model has been implemented within Gadget2 (Springel \& Hernquist 2002) which is based on a modified version of SPH that conserves entropy (energy and angular momentum). Stars are formed according to a probability function defined by the relation between cooling and dynamical times (see also Lia et al. 2001). A standard Salpeter Initial mass function has been assumed. Supernovae II are considered to form from stars with $M>8 M_{\odot}$ and to produce metals according to Woosley \& Weaver (1995) yields. Supernova Ia are assumed to originate from binary systems following the model of Thielemann et al. (1993). We have assumed a random sampling of the lifetime of binary systems in the range 0.1-5 Gyrs.

Chemical elements are distributed within neighbouring gas particles by us-

ing a spline kernel function. In order to estimate the correct environment of stars when SN explode we re-calculate the gaseous neighbours of the stellar particles each time heavy elements are released. In this implementation we have not included the treatment of energy feedback.

We run cosmological tests of $\Lambda$-CDM cosmologies consistent with the concordance model $\left(\Omega_{\Lambda}=0.7, \Omega_{m}=0.3, H_{o}=70 \mathrm{kms}^{-1} \mathrm{Mpc}^{-1}\right)$. The simulations correspond to a volume box of $10 h^{-1} \mathrm{Mpc}(h=0.7)$ with $2 \times 50^{3}$ particles. Three different runs were performed using different cooling functions: solar, primordial and metal-dependent.

\section{Results}

The cosmological simulations are analyzed from a global point of view and then, by identifying the gravitational bounded structures. In Figure 1 (left panel) we show the comoving star formation $\rho_{\mathrm{SFR}}$ and metal production $\rho_{\mathrm{Z}}$ rates for the three runs with solar (solid lines), primordial (dashed lines) and metal-dependent (dotted-dashed lines) cooling functions. We can see that the largest difference is found for the run with fixed solar cooling and for $z \leq 3$. The $\rho_{\mathrm{SFR}}$ of the metaldependent cooling run can be up to $\approx 50 \%$ lower than that of the solar cooling test, and up to $\approx 20 \%$ higher than the primordial cooling run. Similar relations 

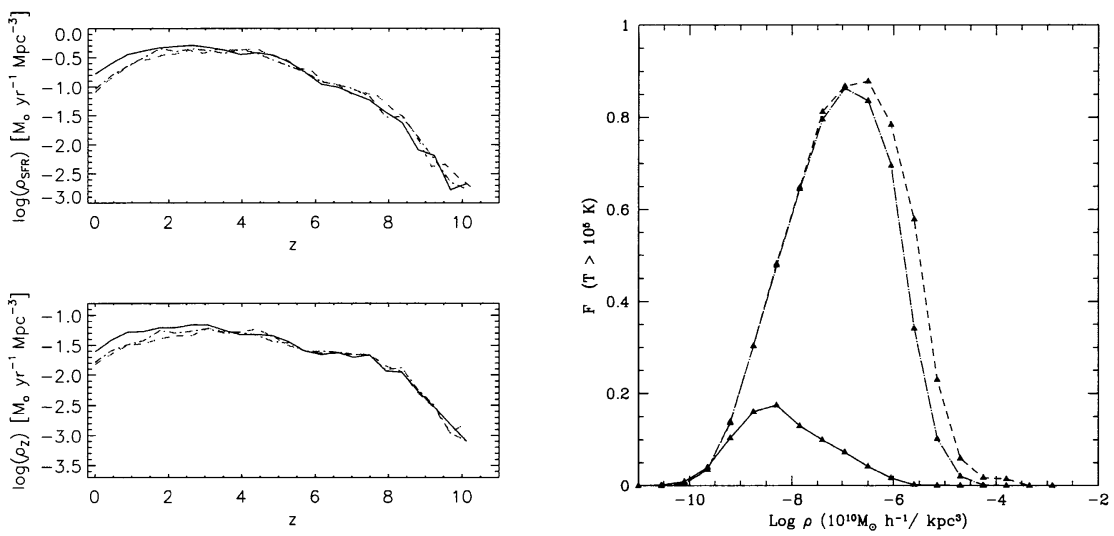

Figure 1. Comoving star formation and metal production rate for three different cosmological runs of the same initial condition, where the gas has been allowed to cool according to solar (solid lines), primordial (dashed lines) and metal dependent (dotted-dashed lines) cooling functions. As can be seen in the left hand panels, the largest differences are found for $z<3$. We found at most a difference of up to $\approx 20 \%$ between the comoving star formation rates of primordial and metal dependent cooling runs.

are found for the metal production rate $\rho_{\mathrm{Z}}$. Figure 1 (right panel) shows the fraction of gas with $T>10^{5} \mathrm{~K}$ for the three runs, with the largest gas fraction associated to the primordial cooling run, as expected. The difference between primordial and metal-dependent cooling are localized in the densest regions.

At $z=0$ we identified galaxy-like objects (GLOs) at their virial radius by combining the friends-of-friends algorithm with a density contrast method in the three simulations (with solar, primordial and metal dependent cooling). We constructed the star formation history of the GLOs and analyzed the chemical properties of their stellar population and interstellar medium. In Figure 2a we show the SFR history of a typical GLO in the three runs. In the small box we have plotted the ratio of the SFR of the solar and metal-dependent cooling to that of the primordial one. We can see that the differences are important for $z<3$, but do not present a regular behavior. This suggests that the non-linear evolution of the structure determines complicated star formation history and chemical patterns that can produce non-linear responses to the characteristics of the cooling functions. Our model will allow us to try to understand the link between the dynamical evolution and the star formation history and chemical patterns, by studying the particular history of evolution of GLOs in different environments.

It was shown by Tissera, Lambas \& Abadi (1997) that the combination of the history of star formation with population synthesis models results in a powerful tool to connect theory with observations. The treatment of the chemical enrichment in the simulations improves the technique since each simulated stellar population has its age and metallicity estimated consistently with the 

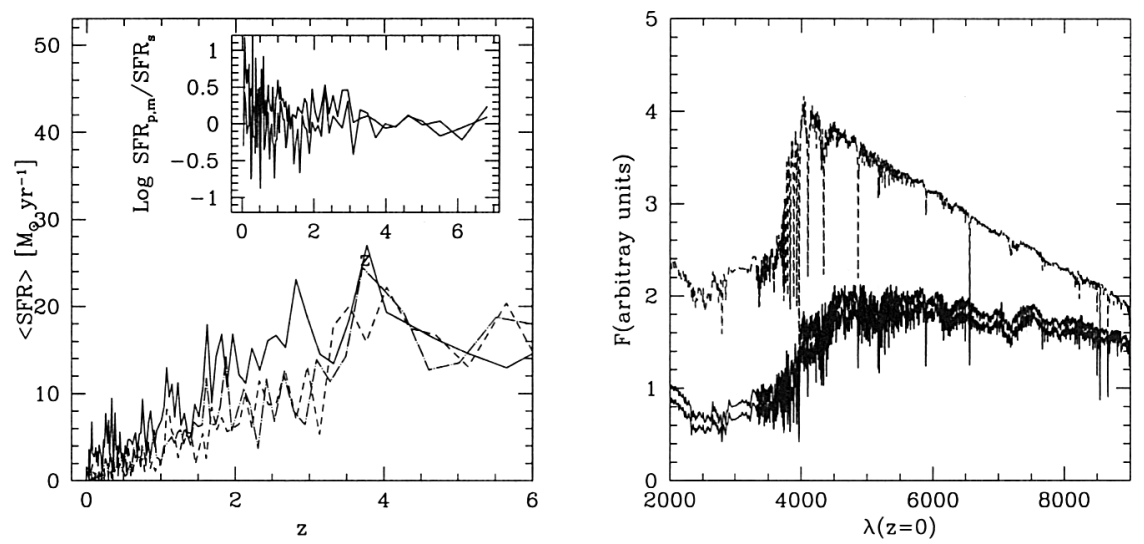

Figure 2. Left panel: Star formation rate history for a typical galaxylike object three different cosmological runs of the same initial condition, where the gas has been allowed to cool according to solar (solid lines), primordial $\left(Z=0.0001 Z_{\odot}\right.$, dashed lines) and metal dependent (dotted-dashed lines) cooling functions. Right panel: Simulated spectra by using the star formation rate history of the metal dependent cooling run and the SED of $\mathrm{BC} 03$ for $Z=0.0001 Z_{\odot}$ (dashed lines), $Z=Z_{\odot}$ (solid lines) and for the corresponding metallicities of the stars (dotted lines).

history of formation in a hierarchical scenario. With this new chemical code, we can also assess the effects of cooling the gas according to its metallicity. For this purpose, we constructed the synthetic spectral distribution of the GLOs but combining the SEDs of Bruzual \& Charlot (2003, BC03) according to the metallicity and age of stars. We also estimated the simulated spectra (SS) of the GLOs by assuming fixed metallicity at $Z=0.0001 Z_{\odot}$ and $Z=Z_{\odot}$ for the SEDs of BC03. In Figure 2b we show the SS for the GLOs of Fig. 2a for the three runs. Note that the SS has been calculated with the same SFR history (Fig. 2a, solid line). From this figure, we can see that assuming a low fixed metallicity leads to a very different energy distribution. For this GLO, the real SS is more similar to that produced by assuming fixed solar metallicity.

We also analyzed the simulated spectra for the same GLO in the three runs (solar, primordial and metal dependent cooling), and estimated the SS by using the full information on age and metallicity. Hence, in this case we will looking at the impact of different gas cooling on the star formation and chemical history through the differences in the energy distribution. In Figure 3 we show the evolution of the SS for the GLO of Figure 2 in the three runs. As can be seen, the GLOs in the solar cooling run exhibit the more luminous spectra since because of the high efficient cooling, a large fraction of stars formed quickly before the gas has time to become enriched. In the case of the corresponding GLO in the runs with primoridal and metal dependent cooling, the interstellar medium has time to get enriched by previous star generations. Hence, in this 

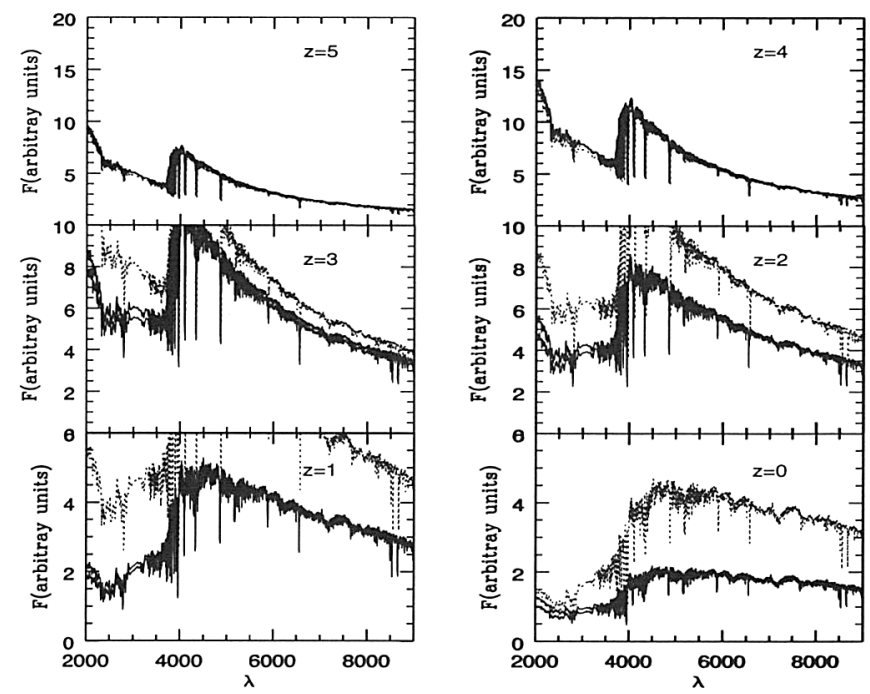

Figure 3. Simulated spectra of the GLO shown in Figure 2 as a function of redshift for the three different cosmological runs of the same initial condition, where the gas has been allowed to cool according to solar (dashed lines), primordial (this solid lines) and metal dependent (thick solid lines) cooling functions.

case, the SS of the GLO metal-dependent cooling run is more similar to that obtained from the primordial one.

\section{Conclusions}

We have incorporated a chemical evolution algorithm within Gadget2 which allows the description of the enrichment of baryons within a cosmological framework. A first analysis shows that in $\Lambda$-CDM scenarios, the comoving star formation rate could be underestimated by up to $\approx 20 \%$ if the primordial cooling function is adopted. However, within individual galactic systems it is complicated to disentangle the effects of cooling the gas consistently with its metallicity owing to the non-linear growth of the structure which affects the star formation, chemical histories and the mixing of metals in a non-linear way. First results show that the star formation history could be either underestimated or overestimated by an order of magnitude depending on the particular history path. We also show the simulated spectra obtained by combining the age and metallicity information of stars in the simulation with the SEDs of Bruzual \& Charlot (2003). Future work will focus on the study of the link between the characteristics of the spectra and history of evolution of the galactic systems. 


\section{Acknowledgments}

We thank Simon White and Volker Springel for useful discussions, and Volker Springel for making Gadget2 available for this work. We acknowledge the support of Fundación Antorchas and DAAD that made this work possible. C. Scannapieco thanks the Alexander von Humboldt Foundation, the Federal Ministry of Education and Research, and the Programme for Investment in the Future (ZIP) of the German Government for partial support. This work was partially funded by CONICET.

\section{References}

Balogh, M.L., et al. 2004, submitted

Barton, E. J., Geller, M. J. \& Kenyon, S. J. 2000, ApJ, 530, 660

Bruzual, G. \& Charlot, S. 2003, MNRAS, 344, 1000

Freeman, K., \& Bland-Hawthorn, J. 2002, ARA\&A, 40, 487

Lambas, D. G., Tissera, P. B., Alonso, M. S., \& Coldwell, G. 2003, MNRAS, in press

Lia, C., Portinari, L, \& Carrari, G. 2002, MNRAS, 339, 821

Mosconi, M. B., Tissera, P. B., Lambas, D. G., \& Cora, S. A. 2001, MNRAS, 325,34

Springel, V., \& Hernquist, L. 2002, MNRAS, 333, 649

Sutherland, R. S., Dopita, M. A. 1993, ApJS, 88, 253

Thielemann, F. K., Nomoto, K., \& Hashimoto, M. 1993, in Origin and Evolution of the Elements, Prantzos, N., Vangoni-Flam, E., Cassé N., eds., p.299

Tissera, P. B., Lambas, D. G., Abadi, M. 1997, MNRAS, 286, 384

Yepes, G., Elizondo, D., \& Ascasibar, Y. 1998, Ap\&SS, 263, 31

Woosley, S. E., \& Weaver, T. A. 1995, ApJS, 101, 181 